\title{
L'assistance au suicide organisée: Quelle place donner au droit?
}

\author{
Benedetta S. Galetti *
}

Durant les dernières décennies, les cas d'assistance au suicide n'ont fait qu'augmenter. Ce développement a été rendu possible essentiellement par l'activité des associations d'aide au suicide et l'interprétation que la jurisprudence et la doctrine ont fait de la réglementation pénale en vigueur. Bien que le droit suisse de l'assistance au suicide est l'un des plus libéraux que l'on connaisse aujourd'hui, les partisans de la mort provoquée demandent depuis des années la reconnaissance d'un véritable droit à la mort. Le cadre qui en résulte de ces faits est préoccupant. Il s'avère essentiel de réaffirmer la nécessité d'une interprétation originaire du droit suisse de l'assistance au suicide, de réaffirmer le droit de chacun au respect de la vie et l'interdiction absolue de nuer et, enfin, de favoriser les soins palliatifs.

I. Introduction

II. La punissabilité de l'assistance au suicide et l'impunité de l'assistance au suicide organisée.

1. L'article $115 \mathrm{CP}$ - Incitation et assistance au suicide....................................78

2. La dépénalisation de l'activité des associations d'aide au suicide..................80

III. La tentative infructueuse d'encadrer l'assistance au suicide organisée et ses conséquences

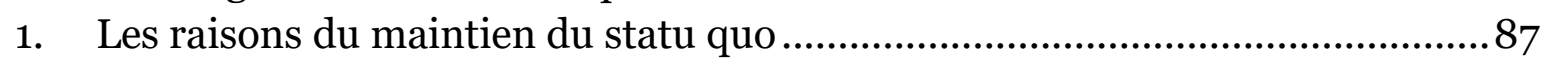

2. Les conséquences du maintien du statut quo ...........................................89

IV. Conclusion

Citation: $\quad$ Benedetta S. Galetti, L'assistance au suicide organisée: Quelle place donner au droit?, dans: sui-generis 2020, S. 76

URL: $\quad$ sui-generis.ch/123

DOI: $\quad$ https://doi.org/10.21257/sg.123

* Benedetta S. Galetti a obtenu un Bachelor en Lettres et Philosophie à l'Université du Sacré Cour de Milan. Elle a ensuite entamé des études de droit et reçu son Bachelor puis son Master à la Faculté de droit de l'Université de Fribourg. Elle a rédigé sa thèse de doctorat, dont le titre est «La mort provoquée - Les fondements, la réglementation légale et des propositions d'alternatives», sous la direction du professeur Henri Torrione. Une version préliminaire de cette contribution a fait l'objet d'une présentation lors du séminaire annuel du programme doctoral en droit de l'Ecole doctorale en droit de la Conférence universitaire de Suisse occidentale (CUSO) du 24 au 25 octobre 2019. L'auteure tient à remercier les Professeurs Franz Werro, Daniel Hürlimann et Andreas Ziegler de leurs remarques.

Ce(tte) œuvre est mise à disposition selon les termes de la Licence Creative Commons Attribution Partage dans les Mêmes Conditions 4.o International. 


\section{Introduction}

1 La technique et les soins médicaux ont réalisé des progrès fulgurants au cours de ces dernières décennies, et l'espérance de vie n'a cessé de s'allonger. Dans le même temps, le risque que les maladies se prolongent a aussi augmenté. Ces situations confrontent les malades à des limites physiques et psychiques souvent extrêmes. La maladie et la déchéance peuvent causer des souffrances telles que la personne qui en souffre en vient à demander la mort.

Le droit suisse de l'assistance au suicide est l'un des plus libéraux que l'on connaisse actuellement. Fruit d'une évolution unique, ses particularités s'expliquent en partie dans l'interprétation que la doctrine et la jurisprudence ont faite de la réglementation pénale en vigueur. La compréhension qui en résulte a permis dans une large mesure aux associations d'aide au suicide de se développer, tout en favorisant une augmentation importante du nombre des suicides assistés. Alors qu'en 2007, le nombre des assistances aux suicides des résidents en Suisse s'élevait à 248 , ce chiffre a augmenté de $274 \%$ pour atteindre 928 en 20161. Ces chiffres ne représentent qu'une partie du nombre des cas. Ils ne comprennent pas celui des personnes étrangères qui, chaque année, viennent en Suisse afin d'y trouver la mort, avec l'appui des associations d'aide au suicide.

3 Le phénomène de l'assistance au suicide organisée préoccupe les milieux politiques. Ceux-ci se sont demandés quelle réponse il convenait d'apporter aux re-

Statistique des causes de décès 2014, Suicide assisté et suicide en Suisse. vendications des partisans du droit à la mort. Le Conseil fédéral a étudié la question pendant plusieurs années. Il n'a toutefois pas réussi à trancher entre la libéralisation de la mort provoquée et son interdiction totale. Il a finalement maintenu le statu quo et appelé au développement des soins palliatifs.

4 Dans la première partie de notre contribution, nous analyserons la clémence dont font preuve les tribunaux dans la répression de l'assistance au suicide. Nous verrons aussi la prolifération des cas de mort provoquée qu'elle entraîne avec l'assistance au suicide organisée (infra II.). Nous rappellerons ensuite la teneur du débat qui a vu le jour en Suisse depuis les années septante. Nous passerons en revue les différentes propositions qui ont vu le jour et celle qui s'est imposée (infra III.).

\section{La punissabilité de l'assistance au suicide et l'impunité de l'assistance au suicide organisée}

5 Le suicide est l'acte par lequel une personne, malade ou en bonne santé, décide librement de mettre un terme à sa vie. Il y a suicide assisté ou accompagnement au suicide lorsque le suicide fait l'objet d'une aide (active ou passive, matérielle ou psychologique), d'un tiers conscient de ce qu'il fait. L'exemple typique d'une telle aide consiste dans le fait de fournir un « cocktail mortel » au suicidant, qui le prend dans ses mains et en avale le contenu ${ }^{2}$. Cette image s'est répandue avec

2 Bien que le plus souvent le produit mortel, à savoir le Natrium Pentobarbital (NaP), soit pris par voie orale, parfois l'administration se fait de manière parentérale ou intraveineuse (Christine Bartsch, Praxis und Probleme des assistierten Suizids in der Schweiz aus rechtsmedizinischer 
l'aide que fournissent les associations d'aide au suicide en Suisse.

6

Le droit pénal ne sanctionne pas le fait de se donner la mort. Il n'en va pas de même pour l'assistance au suicide. Toutefois, ce n'est qu'à certaines conditions que le droit pénal réprime cette assistance.

\section{L'article $115 \mathrm{CP}$ - Incitation et assistance au suicide}

7 L'ordre juridique suisse tout entier protège la vie humaine. En réprimant les atteintes à la vie, le droit pénal revêt au demeurant un rôle central3.

Le suicidant n'encourant aucune sanction pour son acte, celui qui y participe ne saurait encourir une sanction4. C'est le principe de l'accessoriété qui le veut (art. 24 et $25 \mathrm{CP}$ )5. Pour parer à une situation juridique jugée inadéquate, le législateur a cependant choisi d'ériger deux formes de participation en une infraction

Sicht, in: Caroline Welsh/Christoph Ostgathe/ Andreas Frewer/Heiner Bielefeldt [édit], Bielefeld 2016, pp. 203-220, p. 210). Pour une certaine période, et afin d'éviter la nécessité de recourir à un médecin qui soit d'accord à prescrire le $\mathrm{NaP}$, l'association Dignitas proposait le suicide assisté au travers l'inspiration de l'hélium, ensuite abandonné parce que jugé inhumain (Benedetta S. Galetti, La mort provoquée, thèse, Fribourg, en cours de publication, $\mathrm{n}^{\circ} 1691$ ).

3 Ursula Cassani/Marianne Cherbuliez, L'assistance au décès: questions de droit pénal et d'éthique remarques de droit pénal suisse et comparé, in: $\mathrm{Al}-$ berto Bondolfi/Frank Haldemann/Nathalie Maillard (édit.), La mort assistée en arguments, ChêneBourg 2007, pp. 227-266, p. 228.

4 José Hurtado Pozo, Droit pénal - Partie spéciale, Genève/Zurich/Bâle 2009, art. 115 CP, no 215; Christian Schwarzenegger, in: Marcel A. Niggli/ Hans Wiprächtiger (édit.), Basler Kommentar, Strafrecht II - art. 111-392 CP, 4 ème éd., Bâle 2013, art. $115 \mathrm{CP}, \mathrm{n}^{\circ} 1$.

5 Hurtado Pozo (n. 4), n 215; Schwarzenegger (n. 4), $\mathrm{n}^{\circ} 1$. autonome: l'instigation et la complicité au suicide (art. $115 \mathrm{CP})^{6}$.

9 Sur le plan objectif, les éléments constitutifs de l'infraction sont le suicide, consommé ou tenté, et l'incitation ou l'assistance prêtée par l'auteur à la victime qui se suicide.

10 La première condition de l'art. $115 \mathrm{CP}$ est le suicide tenté ou consommé7. Par suicide, on l'a dit, il faut entendre le fait qu'une personne cause personnellement, librement et volontairement sa propre mort. Celle-ci doit donc avoir eu la maîtrise du processus conduisant à sa mort ${ }^{8}$. Cette maîtrise suppose que la personne ait joui d'une pleine capacité de discernement, et qu'elle ait décidé et mis en œuvre son suicide de manière libre et volontaire, en pleine conscience de la situation9.

11 Pour que le suicide ait un caractère personnel, il est nécessaire que la personne ait la maîtrise intellectuelle et physique du geste conduisant à sa mort ${ }^{10}$. Il faut donc qu'elle ait eu la capacité de franchir le dernier pas décisif et qu'elle ait pu changer d'avis jusqu'au dernier instant ${ }^{11}$.

12 C'est au regard de toutes les circonstances du cas qu'il convient d'examiner la capacité de discernement de l'auteur de

$6 \longdiv { \text { Bernard Corboz, Les infractions en droit suisse, } }$

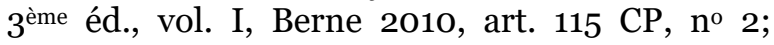
Schwarzenegger (n. 4), no 1 s.

7 Corboz (n. 6), no 3 ; Hurtado Pozo (n. 4), no 234.

8 Hurtado Pozo (n. 4), no 225.

9 Arrêt du Tribunal fédéral 6B_48/2009 du 11 juin 2009, consid. 2.2.1; Hurtado Pozo (n. 4), $\mathrm{n}^{\circ}$ 226; Schwarzenegger (n. 4), nos 2 ss.

10 Hurtado Pozo (n. 4), no 225; Cassani/Cherbuliez (n. 3), p. 234.

11 Schwarzenegger (n. 4), no 11. 
l'acte suicidaire ${ }^{12}$. La vérification de la capacité de discernement se fera en général a posteriori, ce qui inévitablement posera des problèmes de preuve ${ }^{13}$.

13 Encore une fois, non seulement la personne qui se suicide doit avoir la pleine capacité de discernement, mais sa décision doit être libre et volontaire ${ }^{14}$. Le caractère libre et volontaire de la décision peut faire défaut lorsque la personne s'y sent poussée, notamment en raison d'une mauvaise prise en charge médicale, qu'elle se sent abandonnée, ou qu'elle a le sentiment de devenir un poids pour les autres ${ }^{15}$.

14 A l'art. $115 \mathrm{CP}$, le comportement punissable consiste dans une participation. Celle-ci peut revêtir deux formes: l'incitation et l'assistance.

15 Par incitation au suicide, la loi entend un comportement analogue à celui de l'art. $24 \mathrm{CP}^{16}$, lequel consiste dans le fait de convaincre autrui à commettre une infraction ${ }^{17}$. Au sens de l'art. $115 \mathrm{CP}$, l'incitation peut se définir comme le fait de pousser autrui à se suicider ${ }^{18}$. Il appartient toutefois à la personne qui veut

12 Schwarzenegger (n. 4), nos 2 ss.

13 Arrêt du Tribunal fédéral 6B_48/2009 du 11 juin 2009, consid. 2.22.2; Schwarzenegger (n. 4), $\mathrm{n}^{\circ}$ 6; Christian Schwarzenegger, Selbstsüchtige $\mathrm{Be}$ weggründe bei der Verleitung und Behilfe zum Selbstmord, in: Frank Th. Petermann (édit.), Sicherheitsfragen zur Sterbehilfe, Tagungsunterlage zum Anfang 2008 erscheinenden Tagungsband, Saint-Gall 2007, pp. 81-123, p. 88.

14 Arrêt du Tribunal fédéral 6B_48/2009 du 11 juin 2009, consid. 2.2.1; Schwarzenegger (n. 4), no 3 .

15 Galetti (n. 2), n 1190.

16 Arrêt du Tribunal fédéral 6B_48/2009 du 11 juin 2009, consid. 2.2.1; Petra Venetz, Suizidhilfeorganisationen und Strafrecht, thèse, Zurich/Bâle/ Genève 2008, p. 101; Schwarzenegger (n. 4), no 9.

17 Hurtado Pozo (n. 4), $\mathrm{n}^{\circ}$ 1146; Schwarzenegger (n. 4), $\mathrm{n}^{\circ} 9$.

18 Schwarzenegger (n. 4), $\mathrm{n}^{\circ} 9$. se suicider de prendre la décision de mouriri19, sans quoi on ne peut pas admettre qu'elle ait la maîtrise intellectuelle de l'acte.

16 Par l'assistance au suicide, le législateur comprend un comportement analogue à la complicité au sens de l'art. $25 \mathrm{CP}^{20}$. Encore une fois, la terminologie choisie est en lien avec le fait que le suicide auquel elle se rapporte n'est pas une infraction $^{21}$. Dans ce sens, on doit comprendre l'assistance comme l'aide fournie par l'auteur de l'acte suicidaire ${ }^{22}$.

17 L'aide fournie à la personne qui veut mettre fin à ses jours peut revêtir plusieurs formes. Elle peut être à la fois matérielle ou psychologique. Par ailleurs, l'aide peut intervenir avant le suicide, au moment de l'organisation de l'acte ou pendant celui-ci23. La durée de l'assistance n'est pas déterminante ${ }^{24}$. L'exemple classique d'assistance est celui de la fourniture de substances ou de moyens permettant le suicide 25 .

L'incitation et l'assistance au suicide sont deux comportements intentionnels ${ }^{26}$. Le dol éventuel peut toutefois suffire ${ }^{27}$.

19 Schwarzenegger (n. 4), $\mathrm{n}^{\circ} 11$

20 Arrêt du Tribunal fédéral 6B_48/2009 du 11 juin 2009, consid. 2.2.1; Schwarzenegger (n. 4), $\mathrm{n}^{\circ} 10$.

21 Venetz (n. 16), p. 101.

22 Corboz (n. 6), n ${ }^{\circ} 7$.

23 Hurtado Pozo (n. 4), no 224.

24 ATF 92 IV 113 consid 2= JdT 1966 IV 158; ATF 98 IV 83 consid. 2c= JdT 1973 IV 16.

25 Corboz (n. 6), no 7; Schwarzenegger (n. 4), no 11.

26 Schwarzenegger (n. 13), p. 92.

27 Hurtado Pozo (n. 4), no 230; Venetz (n. 16), p. 105. 
$19 \mathrm{Au}$ surplus, et le point est central, l'art. 115 CP s'applique à la condition que l'auteur ait agi avec un mobile égoïste ${ }^{28}$. Il appartient au juge de définir cette notion. On retient en général qu'il y a mobile égoïste lorsque l'auteur agit pour tirer de la mort du suicidé un avantage personnel, direct ou indirect 29 . Cet intérêt peut être de nature matérielle, affective ${ }^{30}$, ou même idéologique ${ }^{31}$. Pour qu'il y ait mobile égoïste, il n'est pas nécessaire que, suite à la mort de la victime, l'avantage se réalise.

Il y a un intérêt matériel lorsque l'auteur agit par cupidité ou dessein de lucre ${ }^{2}$. L'auteur peut vouloir agir par exemple pour toucher un héritage 33 ou obtenir un avantage économique. Celui-ci peut être direct, mais aussi indirect. Le fait d'obtenir un héritage constitue par exemple un avantage direct. Le fait qu'avec la mort d'un concurrent économique, on puisse réaliser une part plus importante de marché est en revanche un avantage indirect. À notre avis, l'emploi qu'on fait de l'avantage économique n'est pas décisif. En d'autres termes, l'emploi honorable d'un avantage économique ne saurait, à lui seul, empêcher l'application de l'art. $115 \mathrm{CP}$.
21 Il y a un intérêt affectif notamment lorsque l'auteur agit par haine, vengeance, rivalité ou méchanceté34.

22 Quant à l'intérêt idéologique35, il existe lorsque l'auteur agit pour promouvoir ses idées. Tel peut être le cas selon nous lorsqu'une personne agit au nom de sa conception de la vie et de la mort, dans l'espoir d'influencer la manière de penser des gens.

\section{La dépénalisation de l'activité des associations d'aide au suicide}

23 La nette augmentation des suicides en Suisse s'explique en partie, à notre avis, avec le développement des associations d'aide au suicide, lequel a pu voir le jour grâce à l'interprétation de l'art. $115 \mathrm{CP}$. De manière générale, la doctrine et la jurisprudence admettent en effet que l'activité de ces associations ne tombe pas sous le coup de cette disposition ${ }^{36}$, le mobile égoïste faisant défaut.

24 À l'heure actuelle, il existe en Suisse sept associations d'aide au suicide. Exit A.D.M.D, Exit Deutsche Schweiz et Dignitas sont les trois plus connues et anciennes. En plus, il y a maintenant LifeCircle, Ex-international, LL Exit et Carpe Diem. Ces associations accompagnent à

34 Corboz (n. 6), no 12; Venetz (n. 16), p. 106.

35 Schwarzenegger (n. 4), no 14; Daniel Häring, Fünf Mythen über Suizidhilfeorganisationen, Jusletter 8 mai 2017, no 56 et 71 .

36 Venetz (n. 16), p. 266 s.; Frank Th. Petermann, Sterbehilfe: Eine terminologische Einführung, in: Frank Th. Petermann (édit.), Sterbehilfe Grundsätzliche und praktische Fragen, SaintGall 2006, pp. 21-44, p. 40; Lorenz Engi, Die «selbstsüchtigen Beweggründe» von Art. 115 StGB im Licht der Normentstehungsgeschichte, Jusletter 4 mai 2009, nº 4 et 19. 
la mort presque toutes les personnes qui décident de recourir au suicide assisté37.

Non seulement elles ont, selon nous, causé une hausse notable du nombre de suicides assistés, mais elles ont aussi favorisé le phénomène du tourisme de la mort. Au cours des dernières décennies, la notoriété et la popularité des associations n'ont fait que s'accroître, et le nombre de leurs membres continue d'augmenter. Les craintes et les critiques qu'elles ont suscitées aussi38.

On a ainsi pu relever qu'aucun contrôle ne s'exerce sur les activités de ces associations, si ce n'est qu'indirectement sous l'angle du droit pénal. Ces associations créent et modifient librement leurs règles statutaires, dont notamment celles qui définissent les conditions d'accès au suicide assisté qui différent souvent d'une association à l'autre39. Elles interviennent sur demande 40 , avec un suivi rapide et une procédure sommaire. En effet, la vérification de la capacité de discernement, la délivrance de l'ordonnance et la mise en place du suicide assisté ne durent en principe pas plus d'un jour41. Les médecins qui interviennent dans la mise en place du suicide assisté font le plus souvent partie de l'association ou, alors, ils en sont très proches. Ils n'ont donc pas l'indépendance requise ${ }^{42}$. Le plus souvent, ils ne connaissent pas les pa-

37 Galetti (n. 2), n 1629.

38 Pour une synthèse, cf. Galetti (n. 2), nos 1626 ss.

39 Galetti (n. 2), no 1806 s.; Bartsch (n. 2), p. 205.

40 Bartsch (n. 2), p. 205.

41 Galetti (n. 2), no 1800; Andreas Brunner, Skizze für ein Gesetz betreffend organisierte Sterbehilfe, in: Christoph Rehmann-Sutter/Alberto Bondolfi/Johannes Fischer/Margrit Leuthold (édit.), Beihilfe zum Suizid in der Schweiz - Beiträge aus Ethik, Recht und Medizin, Berne 2006, pp. 247-253 p. $247 \mathrm{~s}$.

42 Galetti (n. 2), no 1799. tients et pourtant ils délivrent les certificats et l'ordonnance nécessaires de manière rapide et parfois sans même rencontrer les patients43. On ne saurait prétendre dans ces conditions qu'on vérifie de manière sérieuse le caractère libre et volontaire de la demande d'assistance au suicide. À cela s'ajoute que la plupart de ces associations agissent dans l'ombre, sans fournir d'informations par rapport à leur activité. On notera que le prix de l'assistance au suicide dépasse souvent les CHF 10'ooo, et qu'il garantit ainsi un bénéfice clair à ces associations 44 .

\section{a) Une interprétation douteuse de la notion du mobile égoïste}

27 Comme on l'a déjà dit, la doctrine admet en général que l'activité des associations d'aide au suicide ne tombe pas sous le coup de l'art. $115 \mathrm{CP} 45$. Elle retient en effet que le mobile égoïste fait défaut.

28 De la même façon, cette doctrine considère que les organisations d'aide au suicide utilisent les ressources financières dans l'intérêt de leurs associés, et non dans un but commercial. Même lorsque le montant perçu pour un suicide assisté est supérieur aux coûts de l'activité et qu'il permet à l'association de faire un bénéfice, les auteurs considèrent que l'intérêt économique fait défaut, dès lors que le bénéfice reste acquis à l'association aux fins de poursuivre le but idéal qui est

43 Bartsch (n. 2), p. 212.

44 Galetti (n. 2), no 1810; Cela est en outre confirmé par les affirmations de Mariangela Gasperini, présidente de LL Exit, et de Isabelle Scherrer, présidente de Carpe Diem, selon lesquelles, des $€ 10$ 'ooo que leurs associations facturent pour chaque suicide, $€$ 3'50o constituent le bénéfice de l'association (Corriere del Ticino, 9 mars).

45 Schwarzenegger (n. 4), no 14; Petermann (n. 36), p. 40; Engi (n. 36), no 4 et 19. 
le sien ${ }^{46}$. A défaut d'intérêt économique, on retient dans cette approche qu'il n'y a pas de mobile égoïste47.

La même position a été défendue par la Cour cantonale de Bâle-ville qui en 2015 a été appelée à se prononcer sur l'activité de l'association Life-Circle. Elle a considéré que même si l'association, à travers l'accompagnement au suicide, aurait réalisé un bénéfice, il n'aurait pas été possible de retenir contre elle le mobile égoïste pour autant que l'éventuel surplus aurait servi à la réalisation du but de l'association 48. Même si exprimée de manière indirecte, cette même position a été prise par le tribunal de district de Zurich qui en 2018 s'est exprimé sur l'assistance au suicide de plusieurs ressortissantes allemandes, fournie par le fondateur de Dignitas. À noter que même lorsque pour une seule assistance l'association a reçu, à différents titres, plus de CHF 100'OoO et bien que les conditions d'accès à l'assistance au suicide posées par l'ASSM n'avaient manifestement pas été respectées - aspect qui n'a même pas été mis en évidence - le tribunal a acquitté le fondateur. Les raisons résident dans le fait que, suivant le tribunal de district de Zurich qui s'est fondé sur la position de Häring 49 en le citant tout au long de l'arrêt, pour admettre l'existence des motifs égoïstes, il ne suffit pas que ces derniers soient prouvés. Il faut aussi prouver que parmi tous les motifs qui ont poussé l'auteur à

46 Petermann (n. 36), p. 40; Häring (n. 35), no 62.

47 Schwarzenegger (n. 13), p. 118.

48 Arrêt du tribunal de Bâle-Ville S150806102 du 29 octobre 2015, ch. 4.

49 Suivant cet auteur, l'égoïsme commence seulement avec la prédominance de motifs égoïstes (Häring [n. 35], nº 56). son acte, les motifs égoïstes étaient ceux qui ont prévalu50.

3 Le plus souvent, les accompagnants au suicide se font payer une somme d'argent pour chaque cas qu'ils prennent en charge. Cette somme doit servir à couvrir les coûts de la prise en charge et de la mise en œuvre de chaque suicide assisté. On admet en général que lorsque cette somme a pour seul but de rembourser les frais effectifs que l'accompagnant a dû assumer, il n'est pas possible de retenir contre lui un mobile égoïste fondé sur l'intérêt économique51.

31 En pratique, la réalité est moins simple. On ne sait en effet guère comment définir les limites des montants acceptables, la jurisprudence étant par ailleurs pratiquement muette sur la question ${ }^{2}$. Selon certains auteurs, une somme forfaitaire de CHF 350 par suicide assisté constituerait le montant maximal envisageable pour qu'on ne considère pas l'existence d'un mobile égoïste53. Au-delà, il faudrait analyser chaque cas particulier.

32 Moins restrictifs, d'autres auteurs considèrent que, même si l'accompagnant reçoit un montant qui couvre sa perte de gain ou constitue même un véritable salaire, on ne saurait retenir le motif

50 Arrêt du tribunal de district de Uster GG170037I/Mc/Uo2/gp du 30 mai 2018, VIII-X consid. 1 ss. Pour une critique, cf. Daniel Hürlimann, Suizidhilfe ohne Kenntnis des Gesundheitszustands, HSG Focus 3/2019: «Gesundheit» - Dossier.

51 Arrêt du tribunal de Bâle-Ville S150806102 du 29 octobre 2015, ch. 3.2.1; Schwarzenegger (n. 4), $\mathrm{n}^{\circ}$ 14; Venetz (n. 16), p. 266; Häring (n. 35), $\mathrm{n}^{\mathrm{0}} 60$.

52 Schwarzenegger (n. 4), no 14.

53 Cf. Schwarzenegger (n. 13), p. 114; Venetz (n. 16), p. 267. 
égoïste54. On estime ainsi en particulier en relation avec les organisations d'aide au suicide qui assument un nombre considérable de suicides assistés, l'accompagnant aurait droit à ce qu'il gagnerait dans le marché du travail conformément à sa formation 55 .

33 Un avis similaire a été exprimé par le tribunal de district de Zurich, suivant lequel une rémunération annuelle de CHF 130'Ooo versée au président du conseil d'administration correspond à la base normale pour une fonction dirigeante dans les organisations caritatives et il ne serait donc pas possible de retenir le mobile égoïste 56 .

34 On refuse par ailleurs de voir un mobile égoïste, comme on le pourrait, dans l'intérêt idéal de l'aide fournie. Les auteurs retiennent généralement que les associations d'aide au suicide ne poursuivent pas un tel intérêt57.

35 A suivre cette doctrine, le mobile ne serait donc ni idéal ni économique.

\section{b) Une autre compréhension du mobile égoïste et de l'application de l'art. $115 \mathrm{CP}$}

36 Il résulte de la conception qu'on vient de voir que les condamnations en application de l'art. $115 \mathrm{CP}$ sont rares $5^{8}$. À cela

Cf. Petermann (n. 36), p. 40; Venetz (n. 16), p. 268.

55 Cf. Petermann (n. 36), p. 40; Häring (n. 35), $\mathrm{n}^{\text {os }} 67 \mathrm{ss}$.

56 Arrêt du tribunal de district de Uster GG170037I/Mc/Uo2/gp du 30 mai 2018, VIII consid. 3.8.3.

57 Schwarzenegger (n. 4), no 14.

58 Schwarzenegger (n. 13), p. 83. s'ajoute qu'un nombre important de cas ne font pas l'objet d'une dénonciation59. Dans la conception qui est la nôtre, cette situation nous semble pour le moins préoccupante.

37 On le comprendra en rappelant que le législateur a introduit l'art. $115 \mathrm{CP}$ à une époque où les associations d'aide au suicide n'existaient pas et où les discussions sur la mort provoquée n'étaient pas celles qu'on a aujourd'hui ${ }^{60}$. L'introduction de l'art. 115 CP était plutôt fondée sur l'idée «qu'il ne fallait pas punir celui qui agit pour des motifs d'amitié, notamment celui qui agit par pure pitié ou compassion, dans le seul intérêt de la personne qui veut se suicider $» 61$.

38 L'interprétation historique de la norme met en évidence que la dépénalisation de l'incitation et de l'aide au suicide reposait sur le rapport de confiance particulier entre l'auteur et la victime ${ }^{62}$. On comprenait ces actes comme une manifestation de ce lien. Une interprétation téléologique conduit à la même conclusion. Le législateur a justement considéré qu'il n'est pas soutenable de punir celui qui, poussé par des mobiles loyaux et honorables, incite ou aide un ami à se suicider63.

59 Cassani/Cherbuliez (n. 3), p. 230.

60 ATF 136 II 415 consid. 2.3.3 = JdT 211 IV 164; Arrêt du tribunal de district de Uster GG170037I/Mc/Uo2/gp du 30 mai 2018, VII consid. 5.3; Schwarzenegger (n. 13), p. 103; Engi (n. 36), no 16 et 20. Pour un survol sur les discussions et les considérations qui ont mené à l'adoption de l'art 115 CP, cf. Engi (n. 36), nos 6 ss.

61 ATF 136 II 415 consid. 2.3.3 = JdT 211 IV 164; Engi (n. 36), nos 7 ss.

62 Venetz (n. 16), p. 120; Engi (n. 36), nos 9 ss.

63 Schwarzenegger (n. 13), pp. 102 ss; Arrêt du tribunal de district de Uster GG170037I/Mc/Uo2/gp du 30 mai 2018, VII consid. 5.2. 
39 Le fait d'appliquer l'art. $115 \mathrm{CP}$ à l'assistance au suicide organisée, comme le fait le Tribunal fédéral et comme le préconise la doctrine majoritaire, va pour nous clairement à l'encontre de l'interprétation qu'il convient de faire de cette disposition. Les accompagnants interviennent sur appel, en dehors de tout rapport préexistant et de manière routinière. L'intervention a comme unique but celui de fournir les moyens pour mourir.

40 En revanche, favorable à l'application de l'art. $115 \mathrm{CP}$ aux associations d'aide au suicide, Venetz considère qu'il ne se justifie pas d'interpréter la norme de façon restrictive 64 . A notre sens, la question n'est pas là. Au demeurant, les considérations historiques et téléologiques de l'art. 115 CP suffisent à notre avis pour ne pas appliquer cette disposition à l'assistance au suicide organisée.

41 Au contraire de ce que prône la doctrine majoritaire, nous sommes de l'avis qu'on ne peut légitimement exclure systématiquement l'existence du mobile égoïste en relation avec la mise en place des suicides assistés des associations. Comme le remarque un pénaliste français en relation avec la pratique des tribunaux, « [l]e droit pénal est d'interprétation stricte et les tribunaux, quelle que soit la qualification proposée, [doivent refuser] de cautionner des analyses juridiques tentant de détourner le sens de ces incriminations pour les adapter à des situations [...] étrangères à leur objet » 65 .

42 Les montants facturés par les associations d'aide au suicide sont clairement trop élevés pour ne pas reconnaître qu'elles agissent dans un intérêt économique. Comme on l'a dit, le suicide assisté coûte parfois plus de CHF 10'ooo. Il est difficile de croire qu'un suicide assisté puisse effectivement coûter autant. L'argent qui reste à l'association, une fois les frais liés au suicide payé, fonde donc clairement l'existence du mobile égoïste. Que l'association garde l'argent, qu'elle l'investisse ou non n'y change rien. On ne saurait en juger autrement lorsque le montant sert le but idéal de l'association. En effet, ce qui est déterminant à notre avis, c'est que le montant encaissé soit supérieur à celui nécessaire pour couvrir les coûts du suicide assisté.

43 On peut même faire un pas de plus. Comme nous l'avons mis en évidence, on devrait admettre le mobile égoïste même lorsque l'avantage économique escompté est indirect. Tel est le cas lorsqu'avec la publicité qu'elle donne à ses agissements, l'association attend en retour des dons, des legs ou autres. Le mobile égoïste consiste clairement dans le fait d'utiliser le suicide assisté comme un moyen de récolter de l'argent.

44 Cette interprétation de l'art. $115 \mathrm{CP}$ ne contredit au demeurant pas la jurisprudence du Tribunal fédéral sur le dessein de lucre. Pour lui, «le dessein de lucre [...] constitue un critère qualitatif existant déjà lorsque l'auteur recherche un enrichissement particulièrement répréhensible du point de vue moral, parce qu'il met en cause des valeurs relatives à ce qui fait la dignité de la personne et dont la caractéristique est de ne pas être

\footnotetext{
64 Venetz (n. 16), p. 120.

65 Frédéric Archer, Le consentement en droit pénal de la vie humaine, thèse, Paris 2002, $\mathrm{n}^{\circ} 71$.
} 
monnayable ou d'être bafouée lorsqu'elles sont monnayées $» 66$.

Notre interprétation du mobile égoïste trouve au demeurant un appui dans la position qu'avait exprimée le Conseil fédéral, lequel avait considéré que « l'importance et la constance des gains réalisés [par les associations d'aide au suicide], fourni[t] un indice [...] de la présence d'un tel mobile $» 67$. À propos d'une des variantes de modification de l'art. $115 \mathrm{CP}$, qui entendait réprimer le fait pour une organisation d'aide au suicide de recevoir " une prestation appréciable en argent du suicidant ou de ses proches, à l'exception des cotisations de membres et des libéralités versées au moins un an avant le décès ou attribuées dans ce même délai » (cf. art. 115 al. 3 lit. b de l'avant-projet/option 1 discuté en 2009) ${ }^{68}$, le Conseil fédéral avait précisé "qu'une disposition de cette nature ne ferait que concrétiser des obligations découlant déjà du droit en vigueur [...] [qui] permet de réprimer les abus financiers qui pourraient commettre les organisations d'assistance au suicide $» 69$.

En relation avec les personnes actives à l'intérieur des associations, on pourrait selon nous retenir le mobile égoïste à chaque fois que le montant perçu va audelà de la stricte couverture des frais. Lorsque l'assistance au suicide devient une occupation à part entière, il n'est en effet pas possible d'exclure le mobile égoïste. Pour admettre le contraire, il

ATF 113 IV 22 consid. a.

67 Conseil fédéral (CF), Avis du 8 decembre 2006.

68 Avant-projet/option 1 du 28 octobre 2009 pour une Modification du Code pénal.

69 Communiqué de presse de Conseil fédéral, Assistance au suicide: renforcer le droit à l'autodétermination, 29 juin 2011. faudrait que l'activité relève du bénévolat, seuls les frais effectifs justifiant alors une couverture.

47 Par ailleurs, comme on l'a vu, on admet en général que les personnes qui apportent leur assistance au suicide n'agissent pas dans un intérêt idéal.

48 Cette approche peine à convaincre. On ne voit en effet pas pourquoi on ne pourrait pas retenir un tel intérêt, dès lors que ces personnes agissent pour des motifs liés à la satisfaction intérieure, à la misanthropie, à la mise en avant de certaines valeurs et d'un certain altruisme, à la respectabilité, au prestige, au sensationnalisme ou encore au sentiment de pouvoir ${ }^{70}$. On pourrait aussi mettre en cause la publicité ou la propagande que les associations mettent en place ${ }^{71}$.

49 En effet, lorsqu'une association répond à l'appel d'une personne qui désire mourir, elle a le pouvoir de décider si et dans quelle mesure donner suite à ce désir. Selon nous, elle a le pouvoir, à elle seule, de le concrétiser. Elle peut choisir qui doit continuer à vivre et qui peut mourir. Venetz parle à ce propos d'ange de la mort ${ }^{2}$. Nous y verrions plutôt une façon de se prendre pour Dieu ou de s'approprier les pouvoirs qu'on lui prête en exerçant des prérogatives sacrées. Ce n'est pas par hasard si Sobel, ex président de Exit A.D.M.D, dans le documentaire consacré aux activités de l'association dont il assumait la présidence, parle de «sacerdoce $\gg 73$.

70 Venetz (n. 16), p. 271

${ }_{71}$ Schwarzenegger (n. 13), p. 117.

72 Venetz (n. 16), p. 271.

73 Exit, réalisé par Fernand Melgar. 
5o Alors que les proches du défunt s'en prennent souvent à Dieu et à ses représentants, ils épargnent les accompagnateurs des associations d'aide au suicide. En acceptant cette tâche, ces derniers se voient même souvent gratifiés d'une profonde reconnaissance, que ce soit de la part de la personne qui demande à mourir ou de celle des proches. La gratitude va toutefois bien au-delà du cercle restreint du suicidant et de ses familiers. On en a la confirmation dans le fait que les présidents des diverses associations, tout comme les accompagnateurs, se prêtent volontiers aux interviews, aux services télévisés et même aux documentaires.

51 C'est aussi ce qu'a exprimé un arrêt de première instance de 2007 de BâleVille74. Ce tribunal a condamné Peter Baumann, psychiatre de Zurich et fondateur de Verein Suizidhilfe, à trois ans d'emprisonnement dont deux avec sursis. Il l'a considéré comme responsable d'homicide ainsi que d'accompagnement à la mort de plusieurs de ces patients pour mobile égoïste. Le tribunal a souligné que Baumann agissait pour autoglorification et propagande, dans le dessein de se faire connaitre par le public.

On conclura avec une considération en relation avec l'incitation au suicide. Le législateur met sur un pied d'égalité l'incitation et l'aide au suicide. Cette assimilation est douteuse. En effet, au regard de la réalité sociale ainsi que des intérêts sociaux en jeu, elle ne devrait pas aller de soi75. L'aide au suicide se rapporte à une personne déjà déterminée à se suicider, tandis que l'incitation se rap-

74 Arrêt du tribunal de Bâle-Ville 122.10/01 du 21 juillet 2006, ch. 1.2 ss.

75 Cassani/Cherbuliez (n. 3), p. 234. porte à une personne qui n'avait pas la tentation de le faire ou qui, tout au moins, n'était pas déterminée à le faire ${ }^{76}$.

53 À notre sens, on devrait ainsi admettre que la publicité que font les associations d'aide au suicide (spots radio, annonces et articles dans la presse suisse et étrangère) 77 constitue une forme d'incitation qui pourrait tomber sous le coup de l'art. 115 CP. En effet, la publicité faite du suicide assisté est adressée, non pas de manière générale à tous, mais uniquement à ceux qui remplissent les conditions que chaque association a posé pour avoir accès au suicide assisté. C'est précisément dans ce sens que l'incitation est bien adressée à un nombre limité de personne.

54 On remarquera enfin que lorsque l'incitation prive le suicidant de sa liberté, ce n'est pas l'art. 115 CP qu'on devrait appliquer, mais les art. 111 ss CP. La situation dans laquelle l'accompagnant au suicide intervient et que tout est prêt pour l'assistance au suicide qui pourrait éventuellement être filmé, pourrait à cet égard poser des problèmes. Il faudra en effet se demander si l'accompagnant au suicide n'a pas exercé une pression telle qu'il a paralysé la volonté du suicidant.

\section{La tentative infructueuse d'encadrer l'assistance au suicide organisée et ses conséquences}

55 Comme dans les pays voisins, la mort provoquée a suscité et continue à susciter en Suisse un large débat. Les autorités se

76 Cassani/Cherbuliez (n. 3), p. 234.

77 Département fédéral de justice et police (DFJP), Assistance au décès et médecine palliative: la confédération doit-elle légiférer?, 24 avril 2006, p. 10. 
sont interrogées sur l'opportunité de réglementer de manière plus précise l'assistance au suicide organisée ainsi que d'autres formes d'aide à la mort, $\mathrm{y}$ compris l'euthansie. À plusieurs reprises, les pouvoirs publics ont tenté de mettre en place une nouvelle réglementation. Malgré plus d'une dizaine d'interventions parlementaires en une trentaine d'années, la Confédération n' est pas parvenue à mettre sur pied un nouveau cadre légal.

56 À partir de 2002, l'attention politique s'est focalisée sur l'essor des organisations d'aide au suicide. Les autorités politiques se sont rendues compte que le droit ne permettait pas d'éviter les abus de manière efficace ${ }^{78}$. Celles-ci ont alors essayé de concevoir un cadre légal différent qui permettrait de maintenir le recours à l'assistance au suicide tout en contrôlant l'activité des associations qui répondent à ces demandes.

57 En dépit des différentes interventions parlementaires et de plusieurs rapports79, le Conseil fédéral a décidé en 2011 de maintenir le statu quo. Il a conclu que la législation en vigueur était en définitive à même de combattre les éventuels abus. Cela étant, il a réaffirmé sa volonté d'améliorer les soins palliatifs pour prévenir les suicides ${ }^{80}$.

\section{Les raisons du maintien du statu quo}

Il a y plusieurs raisons pour lesquelles le Conseil fédéral a décidé de ne pas donner

78 Schwarzenegger (n. 4), no 20; Galetti (n. 2) $\mathrm{n}^{\text {os }} 1860 \mathrm{ss}$.

79 Schwarzenegger (n. 4), no 22, qui exprime son étonnement à cet égard.

8 Communiqué de presse de Conseil fédéral (n. 69); cf. ég. Schwarzenegger (n. 4), no 21. suite à ceux qui demandaient une nouvelle réglementation sur l'assistance au suicide organisé. En reconnaissant qu'un problème existait, que certaines personnes peuvent demander l'intervention d'une tierce personne afin de mourir, le Conseil fédéral a aussi refusé de reconnaitre un droit à la mort et préféré répondre aux souffrances des personnes en développant les soins palliatifs.

59 Les raisons qui ont fondé le choix du Conseil fédéral sont multiples. La libéralisation de l'assistance au suicide organisée porte en elle le risque d'avaliser cette pratique et de mettre l'État en contradiction avec l'obligation qu'il a de protéger la vie, indépendamment des considérations concernant l'état de santé de la personne. Avec une libéralisation, on devrait ainsi admettre que certaines vies sont dignes d'être vécues alors que d'autres ne le sont pas. Le Conseil fédéral en est conscient: " [c]ette légitimation enverrait à la population le message selon lequel certaines vies sont dignes de protection et d'autres non, ce qui relativiserait l'intangibilité de la vie humaine [...] [et] aurait un effet incitatif ${ }^{81}$. Avec le changement de la loi, on risquerait de donner aux associations d'aide au suicide le pouvoir de décider de la vie et de la mort des gens. En conférant aux individus ce que certains appellent un droit à mourir dignement, on confèrera à quelqu'un d'autre le droit de l'aider à mouririr ${ }^{82}$.

60 Un tel changement risquerait à notre avis de mettre en danger les personnes les plus vulnérables et les plus fragiles. Les personnes âgées ou malades qui vivent des situations d'abandon ou une prise en

81 Communiqué de presse de Conseil fédéral (n. 69).

82 Galetti (n. 2), no 1060. 
charge thérapeutique inadéquate seront les candidats idéaux à l'assistance au suicide. Face à cette nouvelle possibilité, elles pourront faire l'objet de pressions qui leur imposeront de justifier leur désir de continuer à vivre. Le droit de mourir risquerait ainsi de devenir un devoir de mourir 83.

Une telle libéralisation risquerait clairement d'entrainer un accroissement des cas de suicide assisté. En outre, elle ne serait que le point de départ de toute une série de nouvelles revendications. En effet, une telle réglementation constituerait ce qu'on peut définir comme le premier des pas vers l'élargissement des conditions d'accès au suicide assisté, pouvant amener à ouvrir les portes aux personnes âgées fatiguées de vivre, les personnes avec des troubles psychologiques, ou encore les enfants ${ }^{84}$.

62 Avec l'aval étatique qu'une libéralisation de l'assistance au suicide apporterait, on risquerait de mettre en péril un grand nombre de personnes déjà vulnérables, alors que le nombre qui en bénéficierait serait quant à lui très petit. Avec raison, le philosophe australien John Finnis fait remarquer qu'on compromettrait le droit de ne pas être tué pour un nombre beaucoup plus élevé de personnes que celui auquel le droit actuel refuse le droit supposé de mourir85.

63 Face aux personnes vulnérables, la réponse que l'État donne, et par là, la société dans son ensemble, doit être autre que celle de la libéralisation. C'est la conclusion à laquelle selon nous est parvenu le Conseil fédéral en considérant que la priorité doit aller à la prévention du suicide, au travers du développement de soins palliatifs ${ }^{86}$.

64 La question est donc celle de savoir si l'État devrait renoncer à la position ferme qu'il a eue jusqu'à présent, selon laquelle «[t]out être humain a un droit au respect de la vie, y compris de la part de l'État »87. Cette question touche en réalité au «fondement moral de notre société $» 88$. En raison des conséquences qu'une telle renonciation risque de produire, la prudence impose selon nous au législateur de s'abstenir de toute libéralisation. Comme le professeur Torrione, nous dirions à la place que dans « une conception modeste de la fonction du droit, le corps social est constitué par les êtres humains tels qu'ils sont, et ce n'est pas au droit [...] de juger, de la légitimité ou de la qualité de vies humaines $\gg 89$. L'État doit écarter toute autre considération et partir «d'une constatation assez banale »: « [1]a vie, même si elle n'est pas un bienfait, s'impose à chacun de nous comme une donnée primordiale et essentielle ${ }^{90}$.

33 Galetti (n. 2), nos 757 ss.

84 Pour plus de détails, cf. Galetti (n. 2), nos 2021 ss.

85 « $[\mathrm{t}]$ he right not to be killed would be catastrophically nullified for very many more people than the few whose supposed right to die is compromised by present law » (John Finnis, Euthanasia, Morality and Law, Loyola of Los Angeles Law Review 1997-1998/31 pp. 1123-1146, p. 1138).
Communiqué de presse de Conseil fédéral (n. 69). Jean Leonetti, Rapport d'information $n^{\circ} 1287$ réalisé au nom de la mission d'évaluation de la loi no 2005-370 du 22 avril 2005 relative aux droits des malades et à la fin de vie, Paris 28 novembre 2008, audition de Robert Badinter, volume II, p. 570.

88 Leonetti (n. 87), p. 575.

89 Henri Torrione, Le préjudice d'être né. Quelques réflexions à propos de l'ATF 132 III 359, HAVE/REAS 2006/4 pp. 388-398, p. 398.

90 Torrione (n. 89), p. 398. 
Dans la conception ici défendue, l'État doit donc maintenir sa position suivant laquelle « [n]ul ne peut retirer la vie à autrui dans une démocratie $» 91$. On doit donc à notre avis refuser toute modification du cadre légal qui toucherait à ce principe ${ }^{92}$. En effet, même une libéralisation strictement réglementée, qui prévoirait des garde-fous constituerait une atténuation du principe de l'inviolabilité de la vie. Comme on l'a dit lors des discussions parlementaires, "[a]ller plus loin, ce serait aller trop loin $» 93$. Il s'agit donc de choisir en faveur de "l'interdiction absolue de l'homicide, principe qui est l'un des piliers de notre ordre juridique et dont le respect est impérieux $\gg 94$.

A notre avis, il est dès lors juste que les cas exceptionnels suivent le cours ordinaire de la justice95. Il vaut mieux que les juges traitent ces cas, car ils sont les mieux placés pour prendre en considération les particularités de chaque cas et rendre une décision équitable ${ }^{96}$. Les cas sont multiples et le législateur ne pourra jamais tous les appréhender97. C'est la conclusion à laquelle est aussi parvenue le Conseil fédéral qui, en déclarant vouloir maintenir le statu quo, a souligné que «[c]es instruments, flexibles et proches des réalités pratiques, préservent l'équilibre entre le devoir de protection incombant à l'État et le respect de la liberté individuelle $\gg 98$.

\section{Les conséquences du maintien du statut quo}

67 Face à l'impossibilité de réformer les règles qui entourent l'assistance au suicide organisée, le Conseil fédéral a affirmé sa volonté d'améliorer les soins palliatifs. Si on consent à appliquer le droit pénal plus strictement, et donc à notre avis plus justement, comme nous l'avons suggéré plus haut, on ne peut que se rallier à cette volonté.

68 On fait généralement remonter les origines des soins palliatifs à Cicely Saunders. C'est elle qui, en 1967, fonda le St. Christopher's Hospice à Londres, poussée par l'idée que même «quand il n'y a plus rien à faire, [...] [i]l y a toujours quelque chose à faire $» 99$.

69 Lorsqu'on ne peut plus combattre la maladie ni guérir le malade, l'idée de Saunders fut de considérer qu'il est encore possible de le prendre en charge. Dans les établissements de soins palliatifs, on entend traiter les malades en fin de vie pour les pathologies dont ils sont atteints. On leur garantit en plus un soutien psychologique, spirituel et social ${ }^{100}$. Avec le développement d'une prise en charge externe à l'institution, on ne relègue plus la mort à des endroits prédis-

$98 \overline{\text { Communiqué de presse de Conseil fédéral (n. 69). }}$

99 Patrick Verspieren, Face à celui qui meurt - Euthanasie, Acharnement thérapeutique, Accompagnement, Paris 1984, p. 90.

100 Office fédéral de la santé publique (OFSP), Conférence suisse des directrices et directeurs cantonaux de la santé (CSD) et palliative.ch, Cadre général des soins palliatifs en Suisse, Berne 2016, p. 5 . 
posés à cet effet, mais on accepte qu'elle redevienne la « réalité du quotidien »101.

Il n'y a pas une définition de soins palliatifs qui comprenne tous les éléments qui lui sont propres ou qui donne donc entièrement satisfaction. La définition la plus généralement acceptée et régulièrement proposée est celle qu'en a donnée l'OMS en 2002. Elle comprend les soins palliatifs comme l'ensemble des mesures aptes à apaiser les souffrances des personnes atteintes de maladies mortelles et à leur garantir ainsi la meilleure qualité de vie possible jusqu'à la mort ${ }^{102}$.

La médecine palliative part de l'idée selon laquelle la mort est un phénomène naturel ${ }^{103}$. Elle n'entend ni hâter ni différer la mort ${ }^{104}$. Elle a plutôt l'objectif d'accompagner le patient à travers sa maladie, en le préparant physiquement, psychologiquement et spirituellement, à vivre la mort. L'accompagnement à la mort consiste dans une aide emprunte d'humanité, d'empathie, de respect et de considération ${ }^{105}$. Cet accompagnement

$101 \overline{\text { Académie Suisse des Sciences Médicales (ASSM), }}$ Attitude face à la fin de vie et à la mort, Bâle 1 janvier 2018, p. 13 ; L'église catholique dans l'union européenne (COMECE), Opinion of the working group on ethics in research and medicine on palliative care in the European Union, Bruxelles 2016, p. 5 ; Sandra Ester Burkhardt, L'assistance au décès à l'aube du XXIème siècle, thèse, Genève 2011, p. $26 \mathrm{~s}$.

${ }_{102}$ Council of Europe, Recommendation 24 of the Committee of Ministers to member states on the organization of palliative care, Strasbourg 12 novembre 2003, p. 7 et 29; ASSM, Soins palliatifs, Bâle 1 janvier 2013, p. 5 s.; PNR 67, Rapport de synthèse - Fin de vie, Berne 2018, p. 6 et 9 .

103 Council of Europe (n. 102), p. 7 et 29; ASSM (n. 102), p. 6; OFSP/CDS/palliative ch (note 101), p. 10.

104 Council of Europe (n. 102), p. 7 et 29.

105 COMECE (n. 101), p. 12. ne peut se réaliser que dans un rapport de confiance, que seule la communication rend possible ${ }^{106}$. Avec cet accompagnement, on témoigne son amour au patient et que, malgré sa maladie, il mérite encore de vivre sa vie. Les soins palliatifs sont un signe de solidarité de la société envers les membres les plus vulnérables, reconnus et traités conformément à leur dignité107. Les soins palliatifs semblent ainsi offrir une possibilité concrète de mourir dignement.

72 Relativement récente, la médecine palliative a évolué et s'est améliorée de manière rapide et elle continue de le faire. Toutefois, seul un petit nombre de personnes qui en auraient besoin y ont effectivement accès ${ }^{108}$. Malgré les développements de ces dernières années, l'accès aux soins palliatifs reste insuffisant; en outre, il varie d'un canton à l'autre ${ }^{109}$. En raison du vieillissement de la population, la nécessité de soins palliatifs ne pourra au demeurant que s'accentuer ${ }^{110}$.

$106 \overline{\text { ASSM (n. 102), p. } 8 \text { s.; OFSP/CDS, Directives }}$ nationales concernant les soins palliatifs, Berne 2014, p. 12. Sur l'importance de la communication dans le rapport médecin-patient, cf. ég. Benedetta Galetti, Le consentement des mineurs dans le domaine médical, in: Samantha Besson/Yves Mausen/Pascal Pichonnaz (édit.), Le consentement en droit, Genève/Zurich/Bâle 2018, pp. 113-132, pp. 121 ss.

107 Council of Europe (n. 102), p. 7; ASSM (n. 102), p. 8.

108 COMECE (n. 101), p. 16; European Association for Palliative Care (EAPC), La Charte de Prague, Prague 2013, p. 1.

109 DFJP (n. 77), p. 30; Council of Europe (n. 102), p. 2 et 5; PNR 67 (n. 102), p. 14.

110 COMECE (n. 101) p. 16 s.; Council of Europe (n. 102), p. 3 et 7. 
73 De notre point de vue, il est donc urgent de développer et d'améliorer les soins palliatifs ${ }^{111}$. Cette amélioration nécessite une action à plusieurs niveaux. On doit notamment élaborer des politiques de santé appropriées, intégrer la médecine palliative dans le système de santé, renforcer les ressources financières et humaines, développer des institutions spécialisées, faciliter l'accès aux médicaments, améliorer la connaissance des personnes concernées, promouvoir la recherche et améliorer la formation en la matière ${ }^{112}$.

74 De nombreuses personnes partagent ce point de vue. En 2008, Pascal Couchepin, alors chef du Département fédéral de l'intérieur, avait déclaré que la promotion des soins palliatifs constituait l'une de ses priorités. Le 23 octobre 2009, ce département et la Conférence suisse des directrices et directeurs cantonaux de la santé avaient présenté une Stratégie nationale en matière de soins palliatifs 201O-2012. Cette stratégie se concentrait sur les mesures de développement du réseau de soins, de la recherche et de la formation. Le but était d'ancrer les soins palliatifs dans le système de santé suisse afin de garantir un accompagnement adéquat aux patients qui en ont besoin ${ }^{113}$. Les domaines à développer étaient le financement, la sensibilisation de la population, la formation et la recherche. Dès lors que les objectifs ne furent pas atteints, on a poursuivi le projet avec la mise en place de la Stratégie nationale soins palliatifs 2013-2015.

111 COMECE (n. 101) p. 26; Council of Europe (n. 102), p. 6.

112 ASSM (n. 102), p. 18; Council of Europe (n. 102), pp. 10 ss et 53 ss; EAPC (n. 108), pp. 3 ss.

113 OFSP/CDS (n. 106), p. 5.
75 À la fin de ces cinq ans de travail, la Confédération a jugé que de nombreuses mesures avaient vu le jour, notamment dans les domaines des soins, du financement, de la formation, de la sensibilisation, de la recherche et du bénévolat. Elle a estimé que si la stratégie rencontrait un certain succès, de nombreux progrès restaient à faire. C'est pour cette raison qu'en 2015, la Confédération et les cantons ont décidé de créer une plate-forme Soins palliatifs. La plate-forme est axée sur les soins, le financement et la coordination des activités. Elle a notamment pour but de promouvoir des formations universitaires et post-grades, de permettre de maintenir la transmission des connaissances et le travail en réseau, de renforcer la prévention et de réduire les besoins en soins. Il s'agit aussi de limiter les charges relatives au personnel et aux coûts et de réaliser le désir de mourir chez soi, dans un environnement familier.

76 En dépit de la volonté de la Confédération de prévenir l'assistance au suicide organisée au travers du développement de soins palliatifs et des efforts fait pour y parvenir, les soins palliatifs restent aujourd'hui insuffisants. C'est ce qu'affirme encore une fois le Rapport du Programme national de recherche "Fin de vie », présenté en 2018114. Bien que la majorité de la population suisse souhaiterait mourir dans un milieu familial, seul un petit nombre de personnes $\mathrm{y}$ meurt. La plupart des décès survient dans les hôpitaux et dans les EMS. Les raisons principales tiennent à l'insuffisance des soins à domicile ${ }^{115}$. En outre, les proches qui assument la tâche

114 PNR 67 (n. 102), p. 6 et 14.

115 PNR 67 (n. 102), p 14. 
de soins à domicile obtiennent très peu d'aide et de soutien, et ils doivent assumer des coûts très importants. Souvent, «le personnel de santé possède [...] des connaissances insuffisantes en soins palliatifs et ne les propose en conséquence que rarement de manière adéquate $» 116$. Bien évidemment, il s'agit d'un constat qui généralise la situation en Suisse, où des disparités cantonales se maintiennent toutefois. En effet, dans certains cantons les pas qui ont été faits en matière de soins palliatifs sont notables. Tel est notamment le cas dans le canton de Zurich, où presque toutes les personnes qui en font la demande ont un accès adéquat à des soins de qualité ${ }^{117}$.

\section{Conclusion}

77 Pour nous, l'État ne doit pas renoncer à la position qu'il a eue jusqu'à présent. Il doit considérer que « [t]out être humain a un droit au respect de la vie, y compris de la part de l'État ${ }^{118}$. Il ne saurait admettre une brèche au principe selon lequel personne n'a pas le droit de tuer autrui. En raison des conséquences qu'une telle brèche risquerait de produire, la prudence impose au législateur de s'abstenir de libéraliser l'assistance au suicide. Le choix de mettre soi-même fin à sa vie et de solliciter l'aide d'autrui doit rester une question qui relève de l'intimité des relations humaines.

78 Le législateur devrait se garder d'encourager l'assistance au suicide. Une approche contraire mettrait en péril la retenue et la modestie dont le droit doit faire

\footnotetext{
116 PNR 67 (n. 102), p. 16.

117 Dorothee Vögeli, Den Pflegeheimen fehlt spezialisierte Hilfe zur Betreuung der Sterbenden, in: Neue Zürcher Zeitung, 6 janvier 2020, p. 11.

118 Leonetti (n. 87), p. 570.
}

preuve en cette matière et compromettrait le respect de la vie qu'il doit garantir. L'État ne saurait donner aux autorités le pouvoir de venir en aide à ceux qui veulent décider de la mort. Il doit protéger les vies telles qu'elles sont.

79 Une application de l'art. 115 CP fidèle aux conceptions qui ont guidé l'adoption de la règle doit conduire à la répression de l'assistance au suicide organisée telle qu'on la pratique en Suisse. Dans la conception qui est la nôtre, les associations qui assurent cette assistance agissent le plus souvent dans un mobile égoïste, tant au plan économique qu'idéal. L'interprétation contraire qu'en fait la doctrine majoritaire avec la bénédiction de la jurisprudence a transformé de manière préoccupante la Suisse en paradis du tourisme de la mort.

80 Dans le même temps, malgré l'inquiétude que ce tourisme a généré et le débat politique qui s'en est suivi, les pouvoirs publics n'ont pas réussi à changer le cadre légal mis en place. Cette impuissance est un signe encourageant.

81 A ce point, il est en effet préférable non seulement de changer l'interprétation laxiste qu'on fait de l'art. $115 \mathrm{CP}$, mais aussi d'éviter de poursuivre de tenter de libéraliser la pratique de l'assistance au suicide organisé.

82 Plutôt que de favoriser l'aide à la mort, l'État doit protéger les vies telles qu'elles sont. Face aux personnes les plus vulnérables, la réponse de l'État à leur demande de mourir devrait dans toute la mesure du possible favoriser la solidarité de l'ensemble de la collectivité nationale. La voie à suivre est celle du développe- 
ment des soins palliatifs, lesquels restent

insuffisants, malgré les efforts entrepris. 